\title{
Thermodynamic Analysis of Stirling Heat Pump based on Thermoeconomic Optimization Criteria
}

\author{
Liya $X u^{a}$ \\ College of Information and Electronic Engineering, Liming Vocational University, \\ Quanzhou, 362000, People's Republic of China
}

\begin{abstract}
A connectionist investigation of irreversible Stirling heat pump cycles that includes both internal and external irreversibilities together finite heat capacities of external reservoirs was carried out. The thermoeconomic optimization for Stirling heat pump is reported. The heating load per unit total cost for the heat pump is proposed as objective functions for the optimization. The optimum performance parameters which maximize the objective functions are investigated. Since the optimization technique consists of both investment and energy consumption costs, the obtained results are more general and realistic.
\end{abstract}

\section{Introduction}

Throughout thermodynamics cycle approaches of refrigerators and heat pump systems, Stirling cycle is one of the important cycle models with regeneration. According to the theory of classical thermodynamics, the Stirling cycle may possess the condition of perfect regeneration through the use of a reversible regenerator and its theoretical efficiency can approach that of a reversible Carnot cycle. Recently huge amounts of attentions have been made by various Scientists on the addressed cycles. Curzon and Ahlborn were introduced the concept of finite time thermodynamics[1] by performing a new work on Carnot heat engine where as they high lighted the effectiveness of addressed engine that operates at maximum power is calculated with their introduced $\left[\eta_{m}=1-\sqrt{T_{L} / T_{H}}\right.$ ]. It should be noted that the output of their proposed formula is always lower than the routine formula of Carnot $\left[\eta_{C}=1-T_{L} / T_{H}\right]$ while closed more appropriate with the actual efficiencies of operating systems given by them. $\mathrm{Wu}$ has been effectively implemented the theorem of finite time thermodynamics to indicate the optimal performance of the various cycles such as Carnot, Stirling heat pump and Brayton systems for various situations[2,3]. Nowadays, optimum performance parameters of the Stirling

\footnotetext{
a Corresponding author: 13860716115@163.com
}

refrigerators and Stirling heat pumps have been investigated systematically by performing finite time thermodynamics as a function of operating conditions [49]. In this paper, we have presented a general thermoeconomic optimization analysis of irreversible Stirling heat pump with imperfect regenerator and with finite heat capacity of external reservoirs and maximize the thermoeconomic function [10-12].

\section{Stirling heat pump model}

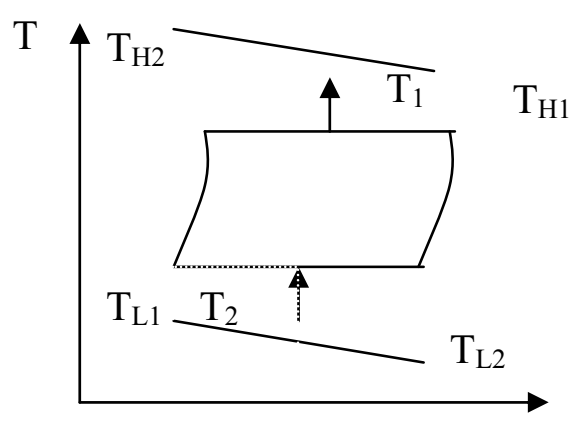

Figure 1. Schematic diagram of the Stirling heat pump As depicted in Fig. 1, the cycle comprises two isochoric and two isothermal processes when the addressed working fluids of the cycle are the ideal gas. The mentioned cycle proximate the expansion stroke of an actual cycle as an isothermal process 1-2 while an irreversible isothermal heat added from a heat source of 
finite heat capacity at temperature $T_{c}$ that the temperature changes in range of $T_{L 1}$ to $T_{L 2}$. Addition of the heat from the regenerator to the addressed working fluid was considered as an isochoric process $2-3$. The compression stroke was considered as isothermal processes 3-4 with an irreversible heat rejection to the heat sink of finite heat capacity at temperature $T_{h}$ that temperature changes in range of $\mathrm{T}_{\mathrm{H} 1}$ to $\mathrm{T}_{\mathrm{H} 2}$ : Lastly, rejection of the heat from the working fluid that comes to the regenerator was considered as an isochoric process 4-1. As expressed previously, the heat transfer processes $1-2$ and 3-4 in an actual cycle must happen throughout finite time. This needs the addressed heat processes would be preferred in the finite temperature difference and consequently, specified as stand hardly irreversible. In addition, the change of entropy through process 3-4 is greater than the change of entropy through process 1-2. So, for each cycle, some heat losses entire the regenerator, as a perfect regeneration needs unlimited regeneration area or time. Therefore, it is necessary to study an actual regenerator. Involving all the above mentioned aspects, the addressed heat pumps approaches turn irreversible. As mentioned previously, the external irreversibility is caused by difference of limited temperature between the external reservoirs and the cycle and on the other hand, regenerative loss.

By defining $\mathrm{Q}_{1}$ and $\mathrm{Q}_{2}$ that represent the value of heat gained at temperature $T_{1}$ from the source and the heat deliver at temperature $T_{2}$ to the sink, respectively, then $[5,6]$ :

$$
\begin{aligned}
& Q_{1}=U_{H} A_{H} \Delta T_{H} t_{1} \\
& =C_{H}\left(T_{H 1}-T_{H 2}\right) t_{1}=n R T_{1} \ln \left(V_{2} / V_{1}\right) \\
& Q_{2}=U_{L} A_{L} \Delta T_{L} t_{2} \\
& =C_{L}\left(T_{L 2}-T_{L 1}\right) t_{2}=n R T_{2} \ln \left(V_{2} / V_{1}\right)
\end{aligned}
$$

Where $U_{H} A_{H}$ and $U_{L} A_{L}$ are the heat transfer coefficient and area products, $C_{H}$ and $C_{L}$ are the heat capacitance rates of the source and sink reservoirs, respectively, and are defined as

$$
\begin{gathered}
\Delta T_{H}=\frac{\left(T_{H 1}-T_{1}\right)-\left(T_{H 2}-T_{1}\right)}{\ln \left[\left(T_{H 1}-T_{1}\right) /\left(T_{H 2}-T_{1}\right)\right]} \\
\Delta T_{L}=\frac{\left(T_{2}-T_{L 1}\right)-\left(T_{2}-T_{L 2}\right)}{\ln \left[\left(T_{2}-T_{L 1}\right) /\left(T_{2}-T_{L 2}\right)\right]}
\end{gathered}
$$

It is also consider the finite heat transfer through the regenerator. Let $\Delta Q_{R}$ is the regenerative heat loss per cycle during the two regeneration processes, which is proportional to the temperature difference of the working fluid as given by

$$
\begin{gathered}
\Delta Q_{R}=n C_{p}\left(1-\eta_{R}\right)\left(T_{1}-T_{2}\right) \\
\tau=\gamma\left(t_{1}+t_{2}\right)
\end{gathered}
$$

Where n is the number of moles, $C_{p}$ the molar specific heat of the working fluid and $\eta_{R}$ the effectiveness of the regenerator. The time of regenerative processes were considered and $\gamma$ is ratio of the time per cycle to the temperature difference of the working fluid. From Eqs.

(1) - (2) we can obtained

$$
\begin{aligned}
& Q_{1}=C_{H} \varepsilon_{H}\left(T_{H 1}-T_{H}\right) t_{1}=C_{H}\left(T_{H 1}-T_{H 2}\right) t_{1} \\
& Q_{2}=C_{L} \varepsilon_{L}\left(T_{L}-T_{L 1}\right) t_{2}=C_{L}\left(T_{L 2}-T_{L 1}\right) t_{2}
\end{aligned}
$$

Where $\varepsilon_{\mathrm{H}}$ and $\varepsilon_{\mathrm{L}}$ are the effectiveness of the hot and cold side of heat exchangers, respectively and are given as $\varepsilon_{H}=1-e^{-\frac{U_{H} A_{H}}{C_{H}}}$ and $\varepsilon_{L}=1-e^{-\frac{U_{L} A_{L}}{C_{L}}}$.

From Eqs. (1) - (8), we can obtain the power input, heating load and the coefficient of performance are, respectively.

$$
\begin{aligned}
R_{H} & =\frac{T_{1}}{\frac{\gamma T_{H}}{C_{H} \varepsilon_{H}\left(T_{H 1}-T_{H}\right)}+\frac{\gamma T_{2}}{C_{L} \varepsilon_{L}\left(T_{L}-T_{L 1}\right)}} \\
C O P_{H} & =\frac{T_{1}}{T_{1}+a\left(T_{1}-T_{2}\right)}
\end{aligned}
$$

Where $\quad a=C_{p}\left(1-\eta_{R}\right) / R \ln \left(V_{2} / V_{1}\right)$. The fundamental optimum relations of the cycle are obtained as

$$
R_{H}=\frac{K T_{H} C O P_{H}\left[1-\left(a+C O P_{H}\right) C O P_{C}^{-1}\right]}{\gamma\left(C O P_{H}+a\right)\left(C O P_{H}+a-1\right)}
$$

Using the above equations, one can obtain the expressions of the important performance parameters of the Stirling heat pump.

\section{Thermoeconomic optimization}

The objective function to be optimized for the heat pump is defined as the heating load per unit total cost, i.e., [911]

$$
F=R_{H} /\left(C_{i}+C_{e}\right)
$$

In which $C_{i}$ and $C_{e}$ indicate annual investment and energy consumption costs, respectively. For the investment cost, we may consider the investment costs of the main system components, which are the heat exchangers and the compressor together with its prime mover. In general, the investment cost of the heat exchangers is assumed to be proportional to the total heat-transfer area. On the other hand, the investment cost 
of the compressor and its driver is assumed to be proportional to its compression capacity. Thus, the annual investment cost of the system can be given as

$$
C_{i}=a_{1}\left(A_{H}+A_{L}\right)
$$

Where the proportionality coefficient for the investment cost of the heat exchangers, a, is equal to the capital recovery factor times investment cost per unit heat transfer area and its dimension is ncu/(year m2). The proportionality coefficient for the investment cost of the compressor and its driver, b, is equal to the capital recovery factor times investment cost per unit power and its dimensionis ncu/(year $\mathrm{kW})$. The unit ncu stands for the national currency unit. The initial investment cost is converted to equivalent yearly payments using the capital recovery factor [9-11]. The annual energy consumption cost is proportional to the heat rate input, i.e.

$$
C_{e}=b P
$$

Where the coefficient, $b$,is equal to the annual operation hours times price per unit energy and its dimension is $\mathrm{ncu} /($ yeark W).Substituting Eqs.(1) to (5) and (9),(10) into Eq.(12), we get

$$
F=R_{H} /\left[a_{1}\left(A_{H}+A_{L}\right)+b P\right]
$$

Using Eqs. (1)-(11) and (15) We obtain

$$
\begin{gathered}
F=\left\{\frac{T_{1}}{\left.\frac{\gamma T_{H}}{C_{H} \varepsilon_{H}\left(T_{H 1}-T_{H}\right)}+\frac{\gamma T_{2}}{C_{L} \varepsilon_{L}\left(T_{L}-T_{L 1}\right)}\right\} /\left\{a_{1}\left(A_{H}+A_{L}\right)\right.}\right. \\
+b\left[\frac{\gamma T_{H}-T_{2}}{\left.\left.\frac{\gamma T_{H}}{C_{H} \varepsilon_{H}\left(T_{H 1}-T_{H}\right)}+\frac{\gamma T_{L}}{C_{L} \varepsilon_{L}\left(T_{L}-T_{L 1}\right)}\right]\right\}}\right.
\end{gathered}
$$

The objective function given in Eq.(16) can be plotted $F / K{ }_{[} K=C_{H} \varepsilon_{H} C_{L} \varepsilon_{L} / \gamma\left(\sqrt{C_{H} \varepsilon_{H}}+\sqrt{C_{L} \varepsilon_{L}}\right)^{2}$ ] with respect to the coefficient of performance of the pump given in Eq.(10), the economical parameter, as shown in Fig.2. As can be seen from the figure, there exists a $C O P_{F}$ which maximizes the objective function.

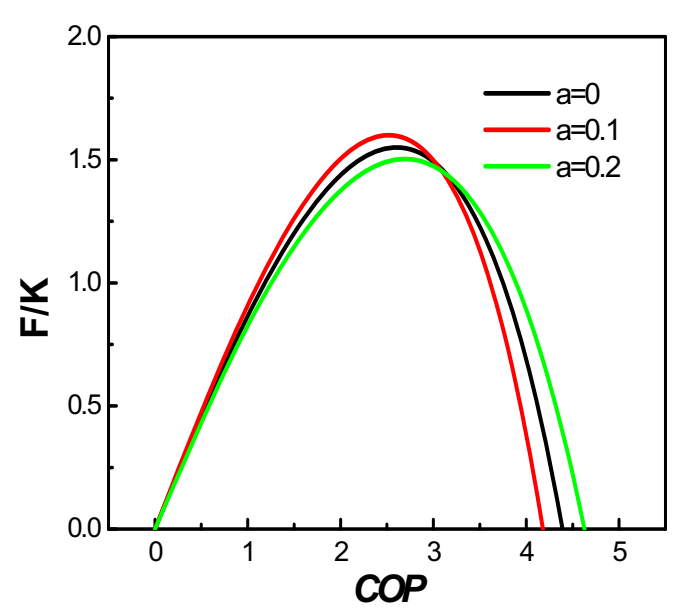

Figure 2 The alteration of the objective function for the heat pump with respect to $C O P$ ( $T_{H 2}=450 \mathrm{~K}, T_{H}=420 \mathrm{~K}, T_{L}=250 \mathrm{~K}, T_{L 2}=200 \mathrm{~K}$, $\left.a_{l}=0.5, b=1, \quad V_{2} / V_{1}=1.2, A_{H}=A_{L}=1 \mathrm{~m}^{2}\right)$.

\section{Conclusions}

In this study, Finite time thermodynamics approach has been carried out to indicate input power, the heating load and the coefficient of performance of the Stirling heat pump. The thermoeconomic optimization for Stirling heat pump is reported. The heating load per unit total cost for the heat pump is proposed as objective functions for the optimization. The optimum performance parameters which maximize the objective functions are investigated.

\section{Acknowledgements}

This work was supported by the project of Science and Technology Research of Quanzhou, Fujian Provice of China (No.2009G10).

\section{References}

1. Curzon F L, Ahlborn B. Amer J Phys, Vol. 43(1975), p.19.

2. Wu C. Int J Ambient Energy, Vol. 14(1993), p.25.

3. Wu C.Energy Convers Manage, Vol. 36(1995), p.7.

4. [4] Chen L, Wu C, Sun F. Energy Convers Manage, Vol. 39(1998), p.113.

5. Chen L, Wu C, Sun F. Energy Convers Manage, Vol. 39( 1998), p.445.

6. Chen L, Wu C, Sun F. Energy Convers Manage, Vol. 39( 1998), p.117.

7. Chen L, Xiaoqin Z, Sun F, Wu C. Appl Energy, Vol. 84(2007), p.78. 
8. Bi Y, Chen L, Sun F. Appl Energy, Vol. 85(2008), p.607.

9. Chen J. Energy Convers Manage, Vol. 39( 1998),p. 1255.

10. Kodal A,Sahin B,Yilmaz T. Energ Convers Manage, Vol. 41(2000), p.607.

11. Kodal A,Sahin B,Oktem A S. Energ Convers Manage, Vol. 41(2000),p.1989.

12. Sahin B,Kodal A,Koyun A. Energ Convers Manage, Vol. 42(2001),p.451. 